\title{
Plasma levels of CGRP and expression of specific microRNAs in blood cells of episodic and chronic migraine subjects: towards the identification of a panel of peripheral biomarkers of migraine?
}

\author{
Rosaria Greco ${ }^{1+}$, Roberto De Icco ${ }^{1,2^{*}+}$ (D) Chiara Demartini ${ }^{1}$, Anna Maria Zanaboni ${ }^{1,2}$, Elena Tumelero ${ }^{1}$,
} Grazia Sances', Marta Allena ${ }^{1}$ and Cristina Tassorelli ${ }^{1,2}$

\begin{abstract}
Background: Migraine can manifest with an episodic or a chronic pattern in a continuum of disease severity. Multiple factors are associated with the progression of the pattern from episodic to chronic. One of the most consistently reported factors is the overuse of medications $(\mathrm{MO})$ for the acute treatment of migraine attacks. The mechanisms through which MO facilitates the transformation of episodic migraine (EM) into chronic migraine (CM) are elusive. In order to provide insights into these mechanisms, the present study aims to identify possible peripheral biomarkers associated with the two forms of migraine, and with the presence of $\mathrm{MO}$.

Methods: We evaluated the plasma levels of calcitonin gene-related peptide (CGRP) and the expression of miR$34 a-5 p$ and miR-382-5p in peripheral blood mononuclear cells of subjects with EM $(n=27)$ or CM-MO $(n=28)$. Subjects in the CM-MO group were also tested 2 months after an in-hospital detoxification protocol.
\end{abstract}

Results: CGRP, miR-382-5p, and miR-34a-5p levels were significantly higher in CM-MO subjects when compared to EM patients ( $p=0.003$ for all comparisons). After correcting for age, sex, and disease duration, miRNAs expression was still significantly associated with migraine phenotype (EM vs. CM-MO: $p=0.014$ for miR-382-5p, $p=0.038$ for miR-34a-5p), while CGRP levels were not $(p=0.115)$. CGRP plasma levels significantly and positively correlated with miR-382-5p (Spearman's rho: 0.491, $p=0.001$ ) and miR-34a-5p (Spearman's rho: 0.303, $p=0.025$ ) in the overall population. In the CM-MO group, detoxification significantly decreased CGRP levels and miRNAs expression ( $p=$ 0.001). When comparing responders and non-responders to the detoxification, the former group $(n=23)$ showed significantly higher levels of CGRP at baseline, and significantly lower expression of miR-382-5p after the detoxification.

(Continued on next page)

\footnotetext{
* Correspondence: rob.deicco@gmail.com

${ }^{\dagger}$ Rosaria Greco and Roberto De Icco contributed equally to this work.

${ }^{1}$ Headache Science \& Neurorehabilitation, IRCCS Mondino Foundation, Pavia, Italy

2Department of Brain and Behavioral Sciences, University of Pavia, Pavia, Italy
}

C C The Author(s). 2020 Open Access This article is licensed under a Creative Commons Attribution 4.0 International License, which permits use, sharing, adaptation, distribution and reproduction in any medium or format, as long as you give appropriate credit to the original author(s) and the source, provide a link to the Creative Commons licence, and indicate if changes were made. The images or other third party material in this article are included in the article's Creative Commons licence, unless indicated otherwise in a credit line to the material. If material is not included in the article's Creative Commons licence and your intended use is not permitted by statutory regulation or exceeds the permitted use, you will need to obtain permission directly from the copyright holder. To view a copy of this licence, visit http://creativecommons.org/licenses/by/4.0/ The Creative Commons Public Domain Dedication waiver (http://creativecommons.org/publicdomain/zero/1.0/) applies to the data made available in this article, unless otherwise stated in a credit line to the data. 
(Continued from previous page)

Conclusions: Our findings identify a potential panel of peripheral markers associated with migraine subtypes and disease severity. CGRP levels as well as miRNAs expression were influenced by MO, and modulated by detoxification in subjects with CM-MO.

Trial registration: The study protocol was registered at www.clinicaltrials.gov (NCT04473976).

Keywords: Migraine, CGRP, MicroRNA, Biomarkers, Pain, Medication overuse, Detoxification, Headache

\section{Background}

Chronic migraine $(\mathrm{CM})$ is a highly disabling condition that frequently manifests as a negative evolution of episodic migraine (EM) in a process that takes place over years [1]. In a recent meta-analysis on the predictors of migraine chronification, a number of monthly headache days $\geq 10$ showed the strongest level of evidence, with depression and low household income being supported by moderate evidence [2]. In the same meta-analysis, medication overuse (MO) was associated to the highest risk ratio in a random-effects model (RR 8.82; 95\% CI, 2.88-27), although the strength of evidence was rated 'very low'due to substantial heterogeneity among studies.

It is likely that CM is the result of the dynamic interaction of multiple co-factors acting on a substrate represented by a more aggressive type of migraine. This hypothesis would explain why as many as $75 \%$ of CM subjects can spontaneously fluctuate between a chronic and an episodic pattern [3]. On the other hand, multiple pieces of evidence show that withdrawal from overused medications induces a clinically meaningful improvement in a large percentage of subjects, with rates of benefit too high to be explained by a simple placebo effect $[4,5]$.

The mechanisms underlying the negative effect of MO in migraine outcome are largely unknown. Multiple neural mechanisms - pronociceptive facilitation, weakened descending pain inhibition and trigeminal hyperexcitability - may be involved $[1,6]$. The loss of diffuse descending inhibition has been demonstrated in a preclinical model of $\mathrm{CM}$ with $\mathrm{MO}$ (CM-MO) [7]. In this frame, it is worth noting that we have recently reported a derangement of the endocannabinoid system in subjects with CM-MO [8], which was more marked when compared with subjects suffering from EM. Interestingly in $\mathrm{CM}-\mathrm{MO}$, the clinical improvement observed after detoxification, namely MO resolution and reduction in headache frequency, is paralleled by the normalization of pain modulation mechanisms and an attenuation of central sensitization, as measured by an increase in the threshold of the nociceptive withdrawal reflex [9]. Preclinical studies show that triptans, the specific acute migraine drugs, induce a condition of hyperalgesia when administered chronically [10]. Thus, it is possible that acute migraine medications, when taken too frequently, may amplify the consequences of nociceptor activation and increase the probability of subsequent migraine attacks, together with the risk of MO.

In this context of multiple concurrent causes, it seems extremely important to investigate in more depth the mechanisms that may be involved in CM-MO. Calcitonin gene-related peptide (CGRP) undoubtedly plays an important, though not exclusive, role in the generation of migraine headache. CGRP receptors are localized in the anatomic sites involved in migraine pathogenesis. CGRP is involved in mast cell degranulation, neurogenic inflammation, and vasodilation [11]. It has been shown that CGRP induces IL-6 gene expression in macrophages by upregulation of circular RNA_007893, a modulator of microRNA-485-5p [12]. microRNAs (miRNAs) are involved in the generation and maintenance of chronic pain and several lines of evidence suggest that specific miRNAs may play a role in migraine pain [13-15]. In a previous clinical study, Andersen and colleagues [15] found an increased expression of miR-34a-5p during migraine attacks, while miR-382-5p levels were increased also in the attack-free phase. In addition, the peripheral expression of miR-34a-5p decreased in the saliva of young migraineurs patients under drug treatment, thus suggesting a possible role in the prediction of the therapeutic response [13]. At present, no reliable individual biomarker of migraine and its subtypes has been identified, though multiple molecules have been proposed and supported by promising results [16].

In order to provide further insights on the mediators involved in migraine pathophysiology and chronification, in this study we assayed the plasma levels of CGRP and the expression of miRNAs in peripheral blood mononuclear cells (PBMCs) of patients with EM and CMMO. As a secondary outcome, we evaluated the changes in CGRP and miRNAs levels after detoxification in the subjects with CM-MO to gather more insights into the mechanisms that are involved in the improvement of migraine pattern following the withdrawal of the overused medications.

\section{Methods}

Study design

This is a cross-sectional observational controlled study with two groups (EM and CM-MO), integrated with a prospective open label interventional trial to assess the 
effect of detoxification in the CM-MO group on the biomarkers of interest. Samples were labeled with numerical codes and all biochemical determinations were performed by researchers who were blind to the diagnosis (RG, CD and AMZ).

\section{Subjects}

Twenty-seven subjects with EM and 28 subjects with CM-MO were consecutively enrolled among patients attending the outpatient clinics of the Headache Science Centre of the IRCCS Mondino Foundation of Pavia (Italy).

Inclusion criteria for EM subjects were: i) diagnosis of migraine without aura according to ICHD-3 criteria [17]; ii) documented history of EM for at least 10 years before enrollment; iii) negative life-time history of CM. Inclusion criteria for $\mathrm{CM}-\mathrm{MO}$ patients were: $\mathrm{i}$ ) diagnosis of $\mathrm{CM}$ and $\mathrm{MO}$ according to ICHD-3 criteria; ii) documented pattern of stable $\mathrm{CM}$ in the 5 years prior to enrollment, without any remission period. This latter point was verified with a high degree of confidence by combining the information obtained from the patients' history with their medical records, including their headache diaries.

At baseline (T0), all patients underwent a visit with a neurologist of the Headache Science Centre during which clinical/demographic data were recorded and inclusion/exclusion criteria were verified. If the criteria were met, subjects underwent peripheral venipuncture for the evaluation of CGRP, miR-382-5p, and miR-34a$5 p$ levels.

Patients with CM-MO were hospitalized for a 7-day standardized detoxification protocol, consisting in abrupt withdrawal of overused drugs associated to intravenous therapy twice daily (08:00 a.m. and 4:00 p.m.) with isotonic $0.9 \% \mathrm{NaCl}$ saline $500 \mathrm{ml}+$ cyanocobalamin $2500 \mathrm{mcg}+$ folic acid $0.70 \mathrm{mg}+$ nicotinamide $12 \mathrm{mg}+$ ascorbic acid $150 \mathrm{mg}+$ sodic glutathione $600 \mathrm{mg}+$ delorazepam $0.5 \mathrm{mg}$ [4]. Two months after hospital discharge (T1), the CM-MO patients returned for a follow-up visit, during which clinical data were recorded and a second venous blood sample obtained from their ante-cubital vein.

At all study points, the patients were tested in an inter-ictal phase, defined as: 1) no headache or headache with non-migraine features and mild intensity (less than 4 on 0 to 10 visual analogue scale) in the $24 \mathrm{~h}$ before blood sampling; and 2) no intake of acute anti-migraine medications in the $24 \mathrm{~h}$ before blood sampling.

The study was approved by the local Ethics Committee (p-20170023682) and all subjects signed a written informed consent upon enrolment, in accordance with the Declaration of Helsinki and existing national ethics regulation. The study protocol was registered at www.clinicaltrials.gov (NCT04473976).

\section{CGRP plasma levels assay}

Blood $(3 \mathrm{ml})$ sampled from the cubital vein of each patient (in the interictal period, between 8:00 a.m. and 12:00 a.m.) was put into a $5 \mathrm{ml}$ lavender tube (BD vacutainer TM, Becton Dickenson, Plymouth, UK). Plasma samples were prepared by centrifugation (2000 $\mathrm{rpm}$ for $15 \mathrm{~min}$ ) and then stored at $-80^{\circ} \mathrm{C}$. Plasma CGRP alpha levels were measured using a commercial enzymelinked immuno-sorbent assay kit (Human $\alpha$-CGRP ELISA kit, Antibodies online, Aachen, DE). Duplicate measurements were performed for each sample and the average value was calculated and considered for the analysis.

\section{Peripheral blood mononuclear cell (PBMC) isolation and miRNA expression}

At the time of enrollment, after overnight fasting, samples of blood $(18 \mathrm{~mL})$ from the cubital vein were collected in sterile tubes from subjects in the interictal period, between 8:00 and 12:00 am.

miRNA expression was evaluated by real-time reverse transcription (RT) PCR in peripheral blood mononuclear cell (PBMCs). PBMCs were isolated immediately after blood collection. Briefly, blood samples were collected within ethylenediamine tetra-acetic acid (EDTA) containing tube and diluted in 1:1 ratio with phosphate buffer saline (PBS) (Sigma Aldrich, Milan, Italy). Then, diluted blood samples were slowly loaded into Ficoll separating solution $(15 \mathrm{ml})$ (Sigma Aldrich, Milan, Italy) and centrifuged at $800 \mathrm{~g}$ for $30 \mathrm{~min}$ at room temperature. PBMCs accumulated as the middle white monolayer, were washed twice in sterile PBS at $300 \mathrm{~g}$ for $15 \mathrm{~min}$. After washing, PBMCs pellet was resuspended in trizol (Bio-Rad, Milan, Italy) and stored at $-80^{\circ} \mathrm{C}$ until use. Total RNA (including all small non-coding RNAs) was extracted from pellets within 2 weeks using the Direct-zol RNA Mini prep plus (Zymo Research from Aurogene, Rome, Italy); then, miRNAs analysis was performed. RNA quality was determined by an optical density (OD) 260/280 ratio $\geq 1.9$ and OD $260 / 230$ ratio $\geq 1.5$ by using a NanoDrop Spectrophotometer (Nanodrop ${ }^{\text {Tw }}$ Thermo Fisher Scientific, Euroclone Milano, Italy). Synthesis of cDNA was performed by using MirXMirna First strand Synthesis (Takara-Diatech Labline, Jesi-Ancona, Italy) and TB Green q-Rt PCR was used (Takara-Diatech, Labline Jesi-Ancona, Italy) to determine expression levels of miRNA-34a-5p and miRNA-382-5p. miRNAs expression was normalized with U6 (a type of small nuclear RNA), used as housekeeping gene. All primers of miRNAs were selected from the Prime 3 software and synthesized by Sigma Aldrich (Milan, Italy). Triplicate reactions were averaged for each miRNA. The cycle threshold $(\mathrm{Ct})$ determination of the two miRNAs ranged from 25 to 28 . U6 expression showed Ct values ranging from 19 to 20 . 
The thermocycling conditions were: $95^{\circ} \mathrm{C}$ for $10 \mathrm{~min}$, and 40 cycles of $15 \mathrm{~s}$ at $95^{\circ} \mathrm{C}$, followed by $1 \mathrm{~min}$ at $60^{\circ} \mathrm{C}$ by a Light Cycler 480 Instrument RT-PCR Detection System (Roche, Milan, Italy). miRNAs levels were calcu-

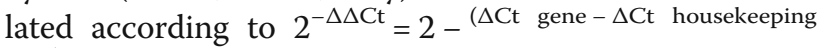
gene) formula by using $\mathrm{Ct}$ values.

\section{Statistical analysis}

Based on a previous study [18], we hypothesized a meaningful difference in peripheral CGRP levels between CM-MO and EM patients of at least $50.0 \pm 60.0 \mathrm{pg} / \mathrm{ml}$ (effect size of 0.981 ). Therefore, for a statistical power of $80 \%$, a risk of $5 \%$ for type 1 errors, and a between groups ratio of 1:1, we calculated a sample size of at least 46 patients (23 subjects with EM and 23 subjects with CM$\mathrm{MO})$. In order to control for possible drop-outs and methodological variability when compared to previous published results [18], we aimed to enroll a population of at least 50 subjects.

The statistical analysis was performed with "R: A language and environment for statistical computing" ( $R$ Foundation for Statistical Computing, Vienna, Austria), Version 1.2.5033, for Windows. Shapiro-Wilk test showed a non-normal distribution of the biochemical variables, therefore we used non-parametric evaluations for all the analysis. Data are presented as "mean \pm standard deviation" for continuous variables, and as "absolute values (percentage)" for categorical variables. Only miRNAs levels are expressed as "mean \pm standard error of the mean", to allow comparison with previous published data [15]. For continuous variables, differences between groups were analyzed using the Mann-Whitney U test, while for categorical variables, statistical analysis was performed with the chi-square test. Correlation analysis was performed by means of the Spearman test. Finally, we performed a logistic regression with the EM and CM-MO as dependent variables, using as covariates the three biochemical dosages as well as other clinical and demographic variables of interest according to the univariate analysis. For all the previously described analyses, the levels of significance were corrected with a Bonferroni method to account for multiple comparison.

To evaluate the role of detoxification in CM-MO patients, we used non-parametric tests for repeated measures [19] with two factors: factor TIME (T0 vs. T1), and factor GROUP (responders vs. non-responders). Patients were qualified as "responders" if they achieved at $\mathrm{T} 1$ a reduction in monthly migraine days of at least $30 \%$ during the previous month, according to the official guidelines for clinical trials in $\mathrm{CM}$ of the International Headache Society [20]. Differences between groups were analyzed using the Mann-Whitney $U$ test or the Kruskal-Wallis test. The levels of significance were further corrected with a Bonferroni method to account for multiple comparisons. For all the performed tests, the level of significance was set at $\alpha=0.050$.

\section{Results \\ Baseline clinical and demographic features of study population}

EM patients ( $n=27 ; 39.2 \pm 8.8$ years; $92.6 \%$ females $)$ reported $6.9 \pm 2.4$ monthly migraine days, and they were taking acute medications on $6.7 \pm 2.2$ days per month. CM-MO patients $(n=28 ; 47.6 \pm 10.9$ years; $85.7 \%$ females) reported $23.6 \pm 5.3$ monthly migraine days, and they were taking acute medications on $23.3 \pm 5.6$ days per month. A percentage of $32.1 \%$ of patients had already undergone at least one detoxification in the past.

CM-MO patients were significantly older than EM patients $(p=0.002)$, and their migraine duration (30.8 \pm 12.1 years) was significantly longer when compared to EM patients $(22.6 \pm 9.7$ years $)(p=0.013)$. Clinical and demographic data are summarized in Table 1.

\section{CGRP and miRNAs profiles in EM and CM-MO}

CGRP plasma levels were higher in the CM-MO group $(393.3 \pm 242.9 \mathrm{pg} / \mathrm{mL})$ when compared to EM patients $(220.4 \pm 83.42 \mathrm{pg} / \mathrm{mL}) \quad(p=0.003)$. The relative expression (RQ) of miR-382-5p in PBMCs was higher in the CM-MO group $(14.6 \pm 3.2)$ when compared to EM patients $(3.3 \pm 0.6)(p=0.003)$. This was also the case for miR-34a-5p expression, which was higher in CM-MO patients $(69.5 \pm 8.5)$ than in EM subjects $(18.4 \pm 4.7)(p=$ 0.003). CGRP plasma levels and miRNAs expression in PBMCs of EM and CM-MO groups are illustrated in Fig. 1.

CGRP plasma levels and relative expression of miR382-5p and miR-34a-5p in PBMCs were not associated to gender, use of preventive therapy or concomitant diagnosis of migraine with aura.

\section{Correlation analysis}

In the overall population, CGRP plasma levels positively correlated with miR-382-5p (Spearman's rho: 0.491, $p=$ 0.001 ) and miR-34a-5p (Spearman's rho: 0.303, $p=$ 0.025 ) expression in the PBMCs. Moreover, CGRP, miR382-5p, and miR-34a-5p levels correlated positively with monthly migraine days, monthly headache days, days of intake of acute drugs and monthly doses of acute drugs ( $p<0.05$ for all comparison). miR-34a-5p levels were also correlated with age (Spearman's rho: 0.321, $p=$ 0.017).

After correction for age, sex, and disease duration using a logistic regression analysis, we found that miRNAs levels were still significantly associated with the migraine phenotype EM/CM-MO ( $p=0.014$ for miR-382$5 \mathrm{p}$, and $p=0.038$ for miR-34a-5p), while CGRP levels were not $(p=0.115)$. The highest effect was recorded for 
Table 1 Clinical and demographic features of study population at baseline

\begin{tabular}{llll}
\hline & EM & CM-MO & p-value \\
\hline $\boldsymbol{n}$ & 27 & 28 & - \\
Age & $39.2 \pm 8.8$ & $24(85.7 \%)$ & 0.002 \\
Sex (female) & $25(92.6 \%)$ & $30.8 \pm 12.1$ & 0.669 \\
Duration of migraine (years) & $22.6 \pm 9.7$ & $12.2 \pm 10.8$ & 0.013 \\
Duration of chronic migraine (years) & - & $8.6 \pm 6.4$ \\
Duration of medication overuse (years) & - & $23.6 \pm 5.3$ \\
Migraine days per month & $6.9 \pm 2.4$ & $26.2 \pm 5.2$ \\
Headache days per month & $7.2 \pm 2.4$ & $23.2 \pm 5.6$ \\
Days of acute drug intake per month & $6.7 \pm 2.2$ & $51.5 \pm 60.5$ \\
Doses of acute drugs per month & $7.2 \pm 2.7$ & $7(25.0 \%)$ \\
Migraine with aura & $5(18.5 \%)$ & $10(35.7 \%)$ \\
Preventive therapy at baseline & $10(37.0 \%)$ & $32.1 \%$ \\
Previous detoxification & - & & - \\
Acute treatment & & $14(50.0 \%)$ \\
$\quad$ NSAID & $13(48.1 \%)$ & $7(25.0 \%)$ \\
$\quad$ Triptan & $8(29.6 \%)$ & $6(21.4 \%)$ \\
Combination & $4(14.8 \%)$ & $1(3.6 \%)$ \\
Politherapy & $2(7.4 \%)$ & 0.001 \\
\hline
\end{tabular}

Legend: $E M$ episodic migraine, CM-MO chronic migraine with medication overuse, NSAID nonsteroidal anti-inflammatory drugs. Continuous variables are presented as mean \pm standard deviation

miR-382-5p, with an odds ratio of 1.794 (95\% confidence interval of 1.124-2.862) for unitary increase in favour of the diagnosis of CM-MO (Table 2). Although a significant correlation was present between CGRP and miRNAs levels, we did not find significant collinearity in the linear regression model.

When the correlation analysis was repeated separately for the EM and CM-MO subgroups, we did not find any significant correlations in the CM-MO group, while in the EM group we obtained a positive and significant correlation between CGRP and miR-382-5p (Spearman's rho: $0.527, p=0.005$ ).

\section{Effects of detoxification from overused drugs in CM-MO patients \\ Clinical outcomes}

All the CM-MO subjects completed the one-week detoxification protocol and returned for the follow-up visit after 2 months (T1). When compared to baseline, at T1 we observed a significant reduction in monthly migraine days $(23.6 \pm 5.3$ vs. $9.6 \pm 7.3 ; p=0.001)$, monthly headache days $(26.9 \pm 5.2$ vs. $13.4 \pm 9.9 ; p=0.001)$, monthly days of intake of acute medications $(23.3 \pm 5.6$ vs. $8.8 \pm$ 7.7; $p=0.001$ ) and monthly doses of acute medications (51.5 \pm 60.5 vs. $11.2 \pm 11.3 ; p=0.001)$.

Eighty-two \% of patients $(n=23)$ qualified as "responders" after 2 months from detoxification, as previously defined, while the remaining 5 patients were "non- responders". Responder and non-responder groups were comparable for clinical and demographic features at baseline. In particular, these groups did not differ at baseline in terms of migraine days (responders: $23.8 \pm$ 5.2; non-responders: $22.8 \pm 6.7 ; p=0.816$ ), headache days (responders: 26.0 \pm 5.1 ; non-responders: $27.2 \pm 6.3$; $p=$ 0.482 ), days of intake of acute medications (responders: $23.5 \pm 5.4$; non-responders: $22.2 \pm 7.4 ; p=0.816)$ and doses of acute medications (responders: $40.5 \pm 31$.3; nonresponders: $102.2 \pm 124.0$; 0.727 ). At $\mathrm{T} 1$, responders reported $7.0 \pm 4.4$ migraine days/month, $11.2 \pm 9.5$ headache days/month, $5.9 \pm 4.5$ days of intake of acute medications/month, and 7.0 \pm 5.5 doses of acute medications/month. Non-responders reported $21.8 \pm 5.1 \mathrm{mi}$ graine days/month, $23.4 \pm 4.0$ headache days/month, $22.0 \pm 4.9$ days of intake of acute medications/month, and $30.4 \pm 11.7$ doses of acute medications/month. At $\mathrm{T} 1$, all the above parameters were significantly different between responders and non-responders, $p<0.020$ ).

\section{CGRP and miRNAs profiles}

At baseline, there were no significant differences between responders and non-responders as regards miR382-5p and miR-34a-5p expression $(p=0.186$, and $p=$ 1.000 , respectively). In contrast, CGRP levels were higher in non-responders when compared to responders $(p=$ 0.036). 


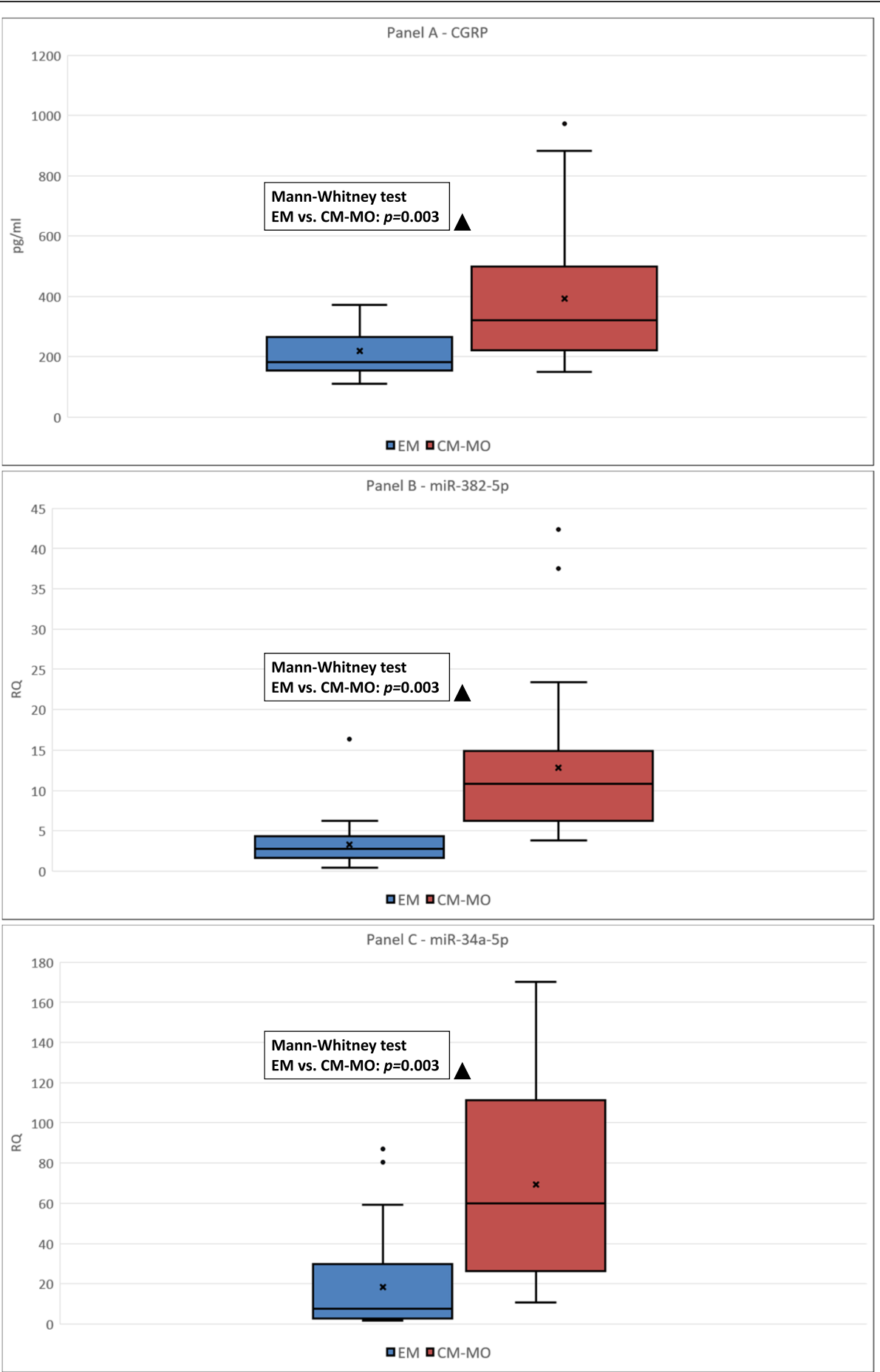

Fig. 1 CGRP plasma levels, and miR-382-5p and miR-34a-5p expression in peripheral blood mononuclear cells of subjects with episodic migraine and chronic migraine with medication overuse. EM: episodic migraine $(n=27)$; CM-MO: chronic migraine with medication overuse $(n=28)$. Panel a: comparison of CGRP between EM and CM-MO subjects. Panel $\mathbf{b}$ : comparison of miR-382-5p between EM and CM-MO subjects. Panel $\mathbf{c}$ : comparison of miR-34a-5p between EM and CM-MO subjects. Box-plots: the distance between the top and bottom of the box represents the interquartile range (IQR: 25th percentile to 75th percentile). The line inside the box represents the median value, while the cross inside the box represents the mean value. The upper and lower whiskers represent the maximum and minimum values respectively (outliers excluded). Black dots above the upper whiskers represent possible outliers (outliers are statistically defined as values outside the 75 th percentile $+1.5^{*} \mathrm{IQR}$ ). $\Delta$ : EM vs. CM-MOH: $p<0.005$ (Mann-Whitney Test with post-hoc Bonferroni's correction). RQ: Relative quantification: $2^{-\Delta \Delta \mathrm{Ct}}=2-{ }^{(\Delta \mathrm{Ct}}$ gene $-\Delta \mathrm{Ct}$ housekeeping gene); Ct: cycle threshold 
Table 2 Results of the logistic regression analysis for dependent variables: EM vs. CM-MO

\begin{tabular}{|c|c|c|c|c|c|c|c|c|}
\hline & B & S.E. & Wald & df & $p$-value & $\operatorname{Exp}(B)$ & \multicolumn{2}{|c|}{ 95\% C.I. for $\operatorname{Exp}(B)$} \\
\hline Age & -0.164 & 2.116 & 1.983 & 1 & 0.159 & 0.849 & 0.676 & 1.066 \\
\hline Sex (male) & -0.150 & 1.538 & 0.010 & 1 & 0.922 & 0.861 & 0.042 & 17.532 \\
\hline Disease duration & 0.221 & 0.131 & 2.845 & 1 & 0.092 & 1.247 & 0.965 & 1.612 \\
\hline CGRP & 0.008 & 0.005 & 2.485 & 1 & 0.115 & 1.008 & 0.998 & 1.017 \\
\hline miR-382-5p & 0.584 & 0.238 & 6.005 & 1 & 0.014 & 1.794 & 1.124 & 2.862 \\
\hline miR-34a-5p & 0.032 & 0.015 & 4.322 & 1 & 0.038 & 1.033 & 1.002 & 1.064 \\
\hline Constant & -6.150 & 2.487 & 6.118 & 1 & 0.013 & 0.002 & - & - \\
\hline
\end{tabular}

Legend: $E M$ episodic migraine, CM-MO chronic migraine with medication overuse. B: coefficients (log-odds units) for the logistic regression equation for predicting the dependent variable (EM vs. CM-MO) from the independent variable. S.E. standard errors associated with the coefficients, Wald Wald chi-square value, $d f$ degrees of freedom. $\operatorname{Exp}(\mathrm{B})$ : odds ratios for the predictors (exponentiation of the coefficients). $95 \%$ C.I. for Exp(B) $95 \%$ confidence intervals for Exp(B)

After detoxification (T1), CGRP levels as well as expression of miR-382-5p, and miR-34a-5p decreased significantly in the overall population (factor TIME "T0 vs. T1": $p=0.001$ for all the three biochemical variables). After detoxification, the pattern of modifications of the three biochemical variables did not differ between responders and non-responders, as suggested by the no significant interaction TIMExGROUP for CGRP $(p=$ $0.542)$, miR-382-5p $(p=0.344)$ and miR-34a-5p ( $p=$ 0.804). CGRP and miRNAs data for CM-MO subjects before and after detoxification are depicted in Fig. 2, and summarized in Table 3.

It is worth noting that, at T1, the levels of CGRP and the expression of miR-34a-5p observed in responders and non-responders were comparable to those of EM patients ( $p=0.472$, and $p=0.248$, respectively), while the expression of miR-382-5p was significantly lower $(p=$ 0.006). This latter difference was mainly explained by the pronounced reduction of miR-382-5p expression recorded in responders (responders vs. EM: $p=0.042$; responders vs. non-responders: $p=0.034$; non-responders vs. EM: $p=1.000)$ (Table 4$)$.

\section{Discussion}

Chronification represents a critical event in the evolution of migraine, as it leads to increased disability and loss in quality of life. Migraine chronification therefore deserves specific scientific attention aimed at identifying the mechanisms and mediators involved.

CGRP is involved in trigeminovascular activation and it is likely to play a role in migraine chronification [21]. CGRP contributes to the release of pro-nociceptive substances from trigeminovascular terminals and to the enhancement of neuronal activity leading to central sensitization [22, 23]. Peripheral levels of CGRP have been proposed as a potential marker of CM, based on the higher values observed interictally, in the absence of symptomatic medications [24]. Plasma CGRP levels may represent a reliable marker of CGRP activity at the receptor level. This hypothesis could explain the high tolerability of the novel anti-CGRP monoclonal antibodies in migraine sufferers. Indeed, CGRP is still present in the plasma of patients chronically treated with erenumab, supporting the idea that a physiological receptor activity is still preserved despite a significant preventive efficacy [25].

Several data on miRNAs expression have shown an association with EM and chronic pain [15, 26]. However, to the best of our knowledge, no study has investigated the role of miR-382-5p and miR-34-5p in CM.

The main finding of this study is that the levels of CGRP and miRNAs were significantly higher in CMMO subjects when compared to EM patients. CGRP and miRNAs levels were also positively correlated with monthly migraine days, monthly headache days, days of intake of acute drugs and doses of acute drugs. It is worth noting that our study population comprises patients with a conspicuous assumption of acute antimigraine drugs, namely with a ratio between migraine days and days of acute drug intake around 1. This is relevant because the almost perfect parallelism between migraine days and days of acute drug intake in our population of CM-MO subjects prevents the possibility to disentangle the role of the respective variables in the observed results. In this frame, it is worth noting that miR-34a-5p expression decreased in the saliva of migraineurs under drug treatment (acute NSAIDs + chronic magnesium), compared with untreated migraineurs [13]. Alterations in miRNAs levels may also reflect reversible alterations in gene expression that occur in response to environmental factors, such as inflammation. miRNAs may play a function in pathological processes, and changes in their regulation are associated with several neurological diseases, including drug addiction [27].

On the other hand, it is important to observe that, after a multivariate analysis with correction for age, sex, and disease duration, miRNAs levels were still significantly associated with migraine phenotype (EM vs. CMMO) ( $p=0.014$ for miR-382-5p, and $p=0.038$ for miR$34 a-5 p)$, while CGRP plasma levels were not $(p=0.115)$. 

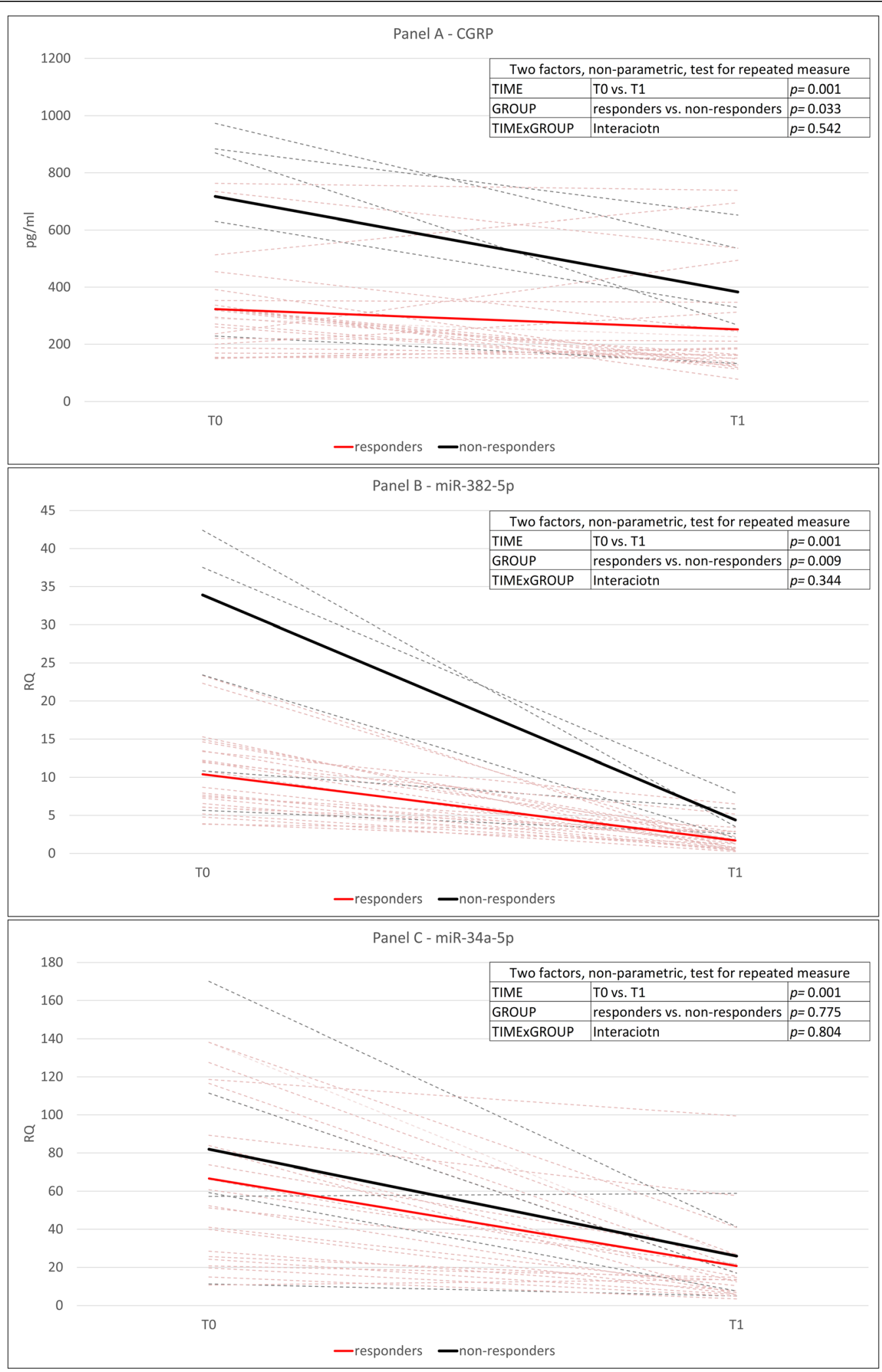

Fig. 2 (See legend on next page.) 
(See figure on previous page.)

Fig. 2 Changes in CGRP plasma levels and miR-382-5p and miR-34a-5p expression in peripheral blood mononuclear cells of subjects with chronic migraine with medication overuse treated with a detoxification protocol. Study population: $n=28 ; 30 \%$ responders: $n=23 ; 30 \%$ non-responders: $n=5$. PBMCs: peripheral blood mononuclear cells. Panel a: changes in CGRP plasma levels after detoxification. Panel $\mathbf{b}$ : changes in miR-382-5p expression in PBMCs after detoxification. Panel $\mathbf{c}$ : changes in miR-34-5p expression in PBMCs after detoxification. The box in the panels highlights the results of statistical analysis performed with two factors, non-parametric test for repeated measures: factor TIME: T0 vs. T1; factor GROUP: 30\% responders vs. non-responders. Dashed lines represent estimates for each patient (red: 30\% responders; black: 30\% non-responders). Continuous lines represent the mean of the group (red: 30\% responders; black: 30\% non-responders). T0: baseline evaluation; T1: 2 months after discharge from in-hospital detoxification. RQ: Relative quantification: $2^{-\Delta \Lambda C t}=2-(\Delta C t$ gene $-\Delta C$ t housekeeping gene); Ct: cycle threshold as defined in methods section

This latter finding, together with the observed reduction in CGRP after the detoxification, suggests that CGRP levels in migraineurs may reflect multiple factors: duration of the disease, clinical phenotype (episodic/ chronic) and intake of acute medications. The conflicting data from the literature on peripheral CGRP levels in EM and CM does not offer solid clues for further elaboration on this finding. Lee et al. found that the interictal serum levels of CGRP were not elevated in CM subjects, and that the use of preventive medications did not influence CGRP levels [28]. The Danish Headache Group [29] reported no difference in CGRP serum levels in CM-MO patients compared with healthy volunteers either before or after detoxification. By contrast, Cernuda-Morollón and co-workers [18] found increased CGRP serum levels in CM women with and without analgesic overuse, compared to EM and healthy controls, thus suggesting a possible pathophysiological mechanism for CGRP in migraine chronification. In line with this hypothesis, CGRP plasma levels were also found significantly elevated in migraineurs requiring preventive therapy [30], suggesting that patients with higher CGRP plasma levels may suffer more frequent migraine attacks. Technical and methodological factors may have contributed to the heterogenicity of results, such as differences in sex distribution, age, specimen source (serum vs plasma), participant selection, timing of blood sample collection and assay methods used (ELISA vs RIA, CGRP alpha isoform vs CGRP beta isoform) [31].
Interestingly, we showed that CGRP plasma levels positively correlated with the peripheral level of both miRNAs. This observation suggests an interaction between CGRP and these signaling molecules. The biological pathways through which the interaction may take place are elusive at this time, but it is possible to hypothesize an inflammation-related mechanism, as suggested by the observation that CGRP induces IL-6 mRNA expression in macrophages by an indirect modulation of miR-485-5p [12].

A further piece of information was provided by the results obtained after detoxification in the CM-MO subjects: the treatment induced a decrease in CGRP, miR382-5p, and miR-34a-5p levels. This finding strongly suggests a role for medication overuse in the biomolecular pathways under investigation. Our findings did not show a clear separation of the effect of detoxification in responder and non-responder groups. Caution is needed in the interpretation of this negative finding because the number of non-responders to detoxification was very small $(n=5)$. However, our findings support the notion that our one-week detoxification protocol and the subsequent reduction of doses of acute medications (a pattern observed also in the non-responder group, though not to a statistically significant level) had an impact on peripheral levels of CGRP and miRNAs, even if it did not translate into a clinically relevant improvement in all the subjects. In this frame, it is interesting to note that a similar pattern of reduction in the levels of the same miRNAs was detected in CM subjects treated with

Table 3 Biochemical data of CM-MO patients before and after detoxification

\begin{tabular}{|c|c|c|c|c|c|c|c|c|c|}
\hline & \multicolumn{3}{|l|}{ T0 } & \multicolumn{3}{|l|}{$\mathrm{T1}$} & \multicolumn{3}{|c|}{ Statistical analysis } \\
\hline & All patients & Responders & Non-responders & All patients & Responders & Non-responders & TIME & GROUP & $\begin{array}{l}\text { TIME } \\
\mathbf{x} \\
\text { GROUP }\end{array}$ \\
\hline$n$ & 28 & 23 & 5 & 28 & 23 & 5 & - & & \\
\hline CGRP $(\mathrm{pg} / \mathrm{ml})$ & $393.3 \pm 242.9$ & $322.9 \pm 164.1$ & $717.1 \pm 301.1$ & $275.2 \pm 251.8$ & $251.8 \pm 383.2$ & $383.2 \pm 208.7$ & 0.001 & 0.033 & 0.542 \\
\hline miR-382-5p (RQ) & $14.6 \pm 3.2$ & $10.4 \pm 1.1$ & $33.9 \pm 15.6$ & $2.2 \pm 0.4$ & $1.7 \pm 0.3$ & $4.4 \pm 1.1$ & 0.001 & 0.009 & 0.344 \\
\hline miR-34a-5p (RQ) & $69.5 \pm 8.5$ & $66.7 \pm 8.8$ & $81.8 \pm 27.2$ & $21.6 \pm 4.0$ & $20.7 \pm 4.4$ & $25.9 \pm 10.4$ & 0.001 & 0.775 & 0.804 \\
\hline
\end{tabular}

Legend: CM-MO chronic migraine with medication overuse. Responders: patients with a reduction of at least $30 \%$ of monthly migraine days after detoxification. T0: baseline evaluation; T1: 2 months after discharge from in-hospital detoxification. CGRP values are presented as mean \pm standard deviation. microRNAs values are presented as "mean \pm standard error of the mean". RQ Relative quantification: $2^{-\Delta \Delta C t}=2-$ ( $\Delta C \mathrm{Ct}$ gene- $\Delta \mathrm{Ct}$ housekeeping gene), $C t$ cycle threshold as defined in methods section. Statistical analysis: non-parametric tests for repeated measures with two factors: factor TIME (T0 vs. T1), and factor GROUP (responders vs. non-responders) 
Table 4 Comparison of biochemical variables between EM and CM-MO patients after detoxification

\begin{tabular}{|c|c|c|c|c|c|c|c|c|}
\hline & EM & CM-MO at $\mathrm{T} 1$ & & & Kruskal- & Post-hoc & & \\
\hline & & All patients & Responders & Non-responders & & $\begin{array}{l}\text { Responders } \\
\text { vs. EM }\end{array}$ & $\begin{array}{l}\text { Responders vs. } \\
\text { non-responders }\end{array}$ & $\begin{array}{l}\text { Non-responders } \\
\text { vs. EM }\end{array}$ \\
\hline$n$ & 27 & 28 & 23 & 5 & & - & & \\
\hline CGRP (pg/ml) & $220.4 \pm 84.4$ & $275.2 \pm 251.8$ & $251.8 \pm 383.2$ & $383.2 \pm 208.7$ & 0.472 & - & - & - \\
\hline miR-382-5p (RQ) & $3.3 \pm 0.63$ & $2.2 \pm 0.4$ & $1.7 \pm 0.3$ & $4.4 \pm 1.1$ & 0.006 & 0.042 & 0.034 & 1.000 \\
\hline miR-34a-5p (RQ) & $18.4 \pm 4.7$ & $21.6 \pm 4.0$ & $20.7 \pm 4.4$ & $25.9 \pm 10.4$ & 0.248 & - & - & - \\
\hline
\end{tabular}

Legend: $E M$ episodic migraine, $C M-M O$ chronic migraine with medication overuse. Responders: patients with a reduction of at least $30 \%$ in monthly migraine days after detoxification. CGRP values are presented as mean \pm standard deviation. microRNAs values are presented as mean \pm standard error of the mean. $R Q R e l a t i v e$

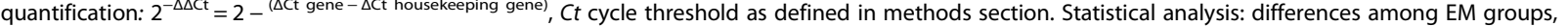
responders, and non-responders patients were analyzed with Kruskal-Wallis test followed by a post-hoc analysis; the levels of significance were further corrected with a Bonferroni method to account for multiple comparison

erenumab regardless of their clinical improvement [32], which suggests that changes in biomolecular pathways implicated in migraine may take place in the absence of a clinical meaningful response. Thus, we hypothesize that, although the levels of miRNAs seem to be strictly related to migraine and its severity, they are not sensitive enough to act as predictors of the clinical improvement [32]. This said, the non-responders to the detoxification are defined by a specific biochemical profile at baseline, characterized by significantly higher levels of CGRP and a tendency toward higher expression of miR-382-5p. This result must be interpreted cautiously due to several reasons: our study was not powered to test this specific hypothesis; the number of non-responders is low $(n=5)$, which does not allow further statistical evaluations, such as intra-group correlation analysis; previous data from literature does not support our finding. Indeed, Munksgaard et al. [29] did not detect significant changes in the peripheral levels of CGRP after detoxification of subjects with MO headache, despite a marked reduction in headache frequency. It must be noted that in their study, the group of subjects included in the post-detoxification evaluation was quite small $(n=10)$ and MO headache was not limited to subjects with migraine as primary headache.

Finally, it is worth noting that the detoxification program reduced CGRP, miR-34a-5p, and miR-382-5p in the CM-MO patients to levels comparable to the EM group, which further underscores the possibility that acute medication overuse actually modifies the biology of migraine.

Some limitations must be acknowledged in the interpretation of our results. Firstly, our study did not include a group of healthy controls. This choice was made to improve the study feasibility, but also because, due to the lack of preliminary data, we first needed to probe the actual existence of an interaction between CGRP and investigated miRNAs. Another limitation is that our study was not primarily powered to test the effects of detoxification and so, although interesting, these results need confirmation in specifically targeted studies. The detoxification protocol indeed proved effective in the large majority of our CM-MO patients, with only 5 out of 28 subjects being non-responders, which ultimately led to numerically imbalanced subgroups and thus prevents generalization of our results.

\section{Conclusions}

The present findings point to a panel of biomolecular markers of migraine subtypes represented by CGRP and two miRNAs. CGRP and miRNAs are associated with each other at the individual level across the migraine spectrum. The peripheral levels of the molecules correlated with the clinical indicators of migraine severity, being higher in CM-MO as compared to EM. Detoxification from medication overuse decreased the peripheral levels of these molecules in the group of subjects with CM-MO. The reported changes provide interesting evidences for a biological effect of detoxification and pave the way for future, larger and specifically targeted, investigations for the characterization and validation of migraine biomarkers, a necessary step for developing personalized therapeutic approaches.

\section{Abbreviations \\ EM: Episodic migraine; CM-MO: Chronic migraine with medication overuse; CGRP: Calcitonin gene-related peptide; PBMC: Peripheral blood mononuclear cell \\ Acknowledgements \\ The authors thank the Research Nurse Team for their precious assistance in all of the activities of the Headache Science Centre of the IRCCS Mondino Foundation.}

\section{Authors' contributions}

Study conception and design: RG, $C$; Enrollment of patients: MA, GS; Acquisition and processing of data: AMZ, CD; Statistical analysis and interpretation of data: RD; Contribution to the first draft of the manuscript: RG, RD; critical revision of results and manuscript: CT, MA, GS. All authors read and approved the final manuscript.

\section{Funding}

This study was supported by grants from the Italian Ministry of Health to IRCCS Mondino Foundation, Pavia, Italy (GR-2016-02363848, and RC20172019). 


\section{Availability of data and materials}

The datasets used and/or analysed during the current study are available from the corresponding author on reasonable request.

\section{Ethics approval and consent to participate}

The study was approved by the local Ethics Committee ( $\left.N^{\circ} \mathrm{p}-20170023682\right)$ and all subjects enrolled signed at T0 a written informed consent, in accordance with the Declaration of Helsinki and existing national ethics regulation.

\section{Consent for publication}

Not applicable.

\section{Competing interests}

$C T$ received honoraria for the participation to advisory boards or for oral presentations from: Allergan, ElectroCore, Eli-Lilly, Novartis, and Teva. CT has no ownership interest and does not own stocks of any pharmaceutical company. CT serves as Chief Section Editor of Frontiers in Neurology-Section Headache Medicine and Facial Pain and on the editorial board of The Journal of Headache and Pain. GS received honoraria for the participation to advisory boards or for oral presentations from: Eli-Lilly and Novartis. The remaining authors have no conflicts of interest.

Received: 18 July 2020 Accepted: 2 October 2020

Published online: 16 October 2020

\section{References}

1. Diener HC, Dodick D, Evers S, Holle D, Jensen RH, Lipton RB, Porreca F Silberstein S, Schwedt T (2019) Pathophysiology, prevention, and treatment of medication overuse headache. Lancet Neurol 18(9):891-902. https://doi. org/10.1016/S1474-4422(19)30146-2

2. Xu J, Kong F, Buse DC (2020) Predictors of episodic migraine transformation to chronic migraine: a systematic review and meta-analysis of observational cohort studies. Cephalalgia. 40(5):503-516. https://doi.org/10.1177/ 0333102419883355

3. Serrano D, Lipton RB, Scher Al, Reed ML, Stewart WBF, Adams AM, Buse DC (2017) Fluctuations in episodic and chronic migraine status over the course of 1 year: implications for diagnosis, treatment and clinical trial design. J HeadachePain 18:101. https://doi.org/10.1186/s10194-017-0787-1

4. Tassorelli C, Jensen R, Allena M, De Icco R, Sances G, Katsarava Z, Lainez M, Leston J, Fadic R, Spadafora S, Pagani M, Nappi G, the COMOESTAS consortium (2014) A consensus protocol for the management of medication-overuse headache: evaluationin a multicentric, multinational study. Cephalalgia. 34(9): 645-655. https://doi.org/10.1177/0333102414521508

5. Rossi P, Faroni JV, Tassorelli C, Nappi G (2013) Advice alone versus structureddetoxification programmes for complicated medication overuse headache (MOH): aprospective, randomized, open-label trial. J Headache Pain 14(1):10. https://doi.org/10.1186/1129-2377-14-10

6. Meng ID, Dodick D, Ossipov MH, Porreca F (2011) Pathophysiology of medicationoveruse headache: insights and hypotheses from preclinical studies. Cephalalgia 31(7):851-860. https://doi.org/10.1177/0333102411402367

7. De Felice M, Ossipov MH, Porreca F (2011) Persistent medication-induced neuraladaptations, descending facilitation, and medication overuse headache. Curr Opin Neurol 24(3):193-196. https://doi.org/10.1097/WCO. 0b013e328346af25

8. Greco R, Demartini C, Zanaboni A, Tumelero E, De Icco R, Sances G, Allena M, Tassorelli C (2020) Peripheral changes of endocannabinoid system components in episodic and chronic migraine patients: a pilot study. Cephalalgia. https://doi.org/10.1177/0333102420949201

9. Perrotta A, Arce-Leal N, Tassorelli C, Gasperi V, Sances G, Blandini F, Serrao M, Bolla M, Pierelli F, Nappi G, MacCarrone M, Sandrini G (2012) Acute reduction of anandamide-hydrolase (FAAH) activity is coupled with a reduction of nociceptive pathways facilitation in medication-overuse headache subjects after withdrawal treatment. Headache. 52:1350-1361

10. De Felice M, Ossipov MH, Wang R, Lai J, Chichorro J, Meng I, Dodick DW, Vanderah TW, Dussor G, Porreca F (2010) Triptan-induced latent sensitization: a possible basis for medication overuse headache. Ann Neurol 67(3):325-337. https://doi.org/10.1002/ana.21897

11. Lambru G, Andreou AP, Guglielmetti M, Martelletti P (2018) Emerging drugs for migraine treatment: an update. Expert Opin Emerg Drugs 23(4):301-318. https://doi.org/10.1080/14728214.2018.1552939
12. Deng T, Yang L, Zheng Z, Li Y, Ren W, Wu C, Guo L (2017) Calcitonin generelated peptide induces IL-6 expression in RAW264.7 macrophages mediated by mmu_circRNA_007893. Mol Med Rep 16(6):9367-9374. https:// doi.org/10.3892/mmr.2017.7779

13. Gallelli L, Siniscalchi A, Carotenuto M, Caroleo MC, Cione E, Guidetti V (2017) microRNAs-based predictor factor in patients with migraineischemic stroke. Microrna. 6(1):17-21. https://doi.org/10.2174/ 2211536606666170104130101

14. Gazerani P (2019) Current evidence on potential uses of MicroRNA biomarkers for migraine: from diagnosis to treatment. Mol Diagn Ther 23(6): 681-694. https://doi.org/10.1007/s40291-019-00428-8

15. Andersen HH, Duroux M, Gazerani P (2016) Serum MicroRNA signatures in migraineurs during attacks and in pain-free periods. Mol Neurobiol 53(3): 1494-1500. https://doi.org/10.1007/s1 2035-015-9106-5

16. Ferroni P, Barbanti P, Spila A, Fratangeli F, Aurilia C, Fofi L, Egeo G, Guadagn F (2019) Circulating biomarkers in migraine: new opportunities for precision medicine. Curr Med Chem 26(34):6191-6206

17. Headache Classification Committee of the International Headache Society (IHS) (2018) The International Classification of Headache Disorders, 3rd edition. Cephalalgia 38:1-211

18. Cernuda-Morollón E, Larrosa D, Ramón C, Vega J, Martínez-Camblor P, Pascual I (2013) Interictal increase of CGRP levels in peripheral blood as a biomarker for chronic migraine. Neurology. 81(14):1191-1196. https://doi. org/10.1212/WNL.0b013e3182a6cb72

19. Noguchi K, Gel YR, Brunner E, Konietschke F (2012) nparLD : an R software package for the nonparametric analysis of longitudinal data in factorial experiments. J Stat Softw 50(12):1-23. https://doi.org/10.18637/ jss.v050.i12

20. Tassorelli C, Diener H-C, Dodick DW et al (2018) Guidelines of the international headache society for controlled trials of preventive treatment of chronic migraine in adults. Cephalalgia. 38(5):815-832. https://doi.org/10. 1177/0333102418758283

21. Russo AF (2015) Calcitonin gene-related peptide (CGRP): a new target for migraine. Annu Rev Pharmacol Toxicol 55:533-552. https://doi.org/10.1146/ annurev-pharmtox-010814-124701

22. Edvinsson JCA, Viganò A, Alekseeva A, Alieva E, Arruda R, De Luca C, D'Ettore N, Frattale I, Kurnukhina M, Macerola N, Malenkova E, Maiorova M, Novikova A, Řehulka P, Rapaccini V, Roshchina O, Vanderschueren G, Zvaune L, Andreou AP, Haanes KA, European Headache Federation School of Advanced Studies (EHF-SAS) (2020) The fifth cranial nerve in headaches. J Headache Pain 21(1):65. https://doi.org/10.1186/s10194020-01134-1

23. Messlinger K (2018) The big CGRP flood - sources, sinks and signalling sites in the trigeminovascular system. J Headache Pain 19(1):22. https://doi.org/ 10.1186/s10194-018-0848-0

24. Ramón C, Cernuda-Morollón E, Pascual J (2017) Calcitonin gene-related peptide peripheral blood as a biomarker for migraine. Curr Opin Neurol 30(3):281-286. https://doi.org/10.1097/WCO.0000000000000440

25. Tringali G, Navarra P (2019) Anti-CGRP and anti-CGRP receptor monoclonal antibodies as antimigraine agents. Potential differences in safety profile postulated on a pathophysiological basis. Peptides. 116:16-21. https://doi. org/10.1016/j.peptides.2019.04.012

26. Andersen HH, Duroux M, Gazerani P (2014) MicroRNAs as modulators and biomarkers of inflammatory and neuropathic pain conditions. Neurobiol Dis 71:159-168. https://doi.org/10.1016/j.nbd.2014.08.003

27. Li MD, van der Vaart AD (2011) MicroRNAs in addiction: adaptation's middlemen? Mol Psychiatry 16(12):1159-1168. https://doi.org/10.1038/mp.2011.58

28. Lee MJ, Lee SY, Cho S, Kang ES, Chung CS (2018) Feasibility of serum CGRP measurement as a biomarker of chronic migraine: a critical reappraisal. J Headache Pain 19(1):53. https://doi.org/10.1186/s10194018-0883-x

29. Munksgaard SB, Ertsey C, Frandsen E, Bendtsen L, Tekes K, Jensen RH (2019) Circulating nociceptin and CGRP in medication-overuse headache. Acta Neurol Scand 139(3):269-275. https://doi.org/10.1111/ane.13053

30. Fan PC, Kuo PH, Lee MT, Chang SH, Chiou LC (2019) Plasma calcitonin gene-related peptide: a potential biomarker for diagnosis and therapeutic responses in pediatric migraine. Front Neurol 10:10. https://doi.org/10.3389/ fneur.2019.00010

31. Frederiksen SD, Bekker-Nielsen Dunbar M, Snoer AH, Deen M, Edvinsson L (2020) Serotonin and neuropeptides in blood from episodic and chronic migraine and cluster headache patients in case-control and case-crossover 
settings: a systematic review and meta-analysis. Headache. 15. https://doi. org/10.1111/head.13802

32. De Icco R, Fiamingo G, Greco R, Bottiroli S, Demartini C, Zanaboni AM,

Allena M, Guaschino E, Martinelli D, Putorti A, Grillo V, Sances G, Tassorelli C

(2020) Neurophysiological and biomolecular effects of erenumab in chronic

migraine: an open label study. Cephalalgia. 26:333102420942230.

https://doi.org/10.1177/0333102420942230

\section{Publisher's Note}

Springer Nature remains neutral with regard to jurisdictional claims in published maps and institutional affiliations.

Ready to submit your research? Choose BMC and benefit from:

- fast, convenient online submission

- thorough peer review by experienced researchers in your field

- rapid publication on acceptance

- support for research data, including large and complex data types

- gold Open Access which fosters wider collaboration and increased citations

- maximum visibility for your research: over $100 \mathrm{M}$ website views per year

At $\mathrm{BMC}$, research is always in progress.

Learn more biomedcentral.com/submissions 